\title{
Practice-Based Teacher Education with ELK: A Role-Playing Simulation for Eliciting Learner Knowledge
}

\author{
Xu Wang ${ }^{1}$, Meredith Thompson ${ }^{2}$, Kexin Yang ${ }^{1}$, Dan Roy ${ }^{2}$ \\ Kenneth Koedinger ${ }^{1}$, Carolyn Rose ${ }^{1}$, Justin Reich ${ }^{2}$ \\ ${ }^{1}$ Human-Computer Interaction Institute, Carnegie Mellon University, Pittsburgh, PA, USA \\ ${ }^{2}$ Teaching Systems Lab, Massachusetts Institute of Technology, Cambridge, MA, USA \\ \{xuwang, kexiny, kk1u, cp3a\}@andrew.cmu.edu, \{mtknight, danroy, jreich\}@mit.edu
}

\begin{abstract}
Compared to other helping professions, teacher training typically lacks sufficient opportunities for novices to practice new skills. When teachers learn, they listen to people talk about teaching, or talk about teaching themselves, but they very rarely do the work of teaching. Games and simulations offer a promising framework to advance practice-based professional training for complex skills such as teaching. In this work, we built a role-playing simulation ELK to help teachers develop effective questioning strategies to elicit learner prior knowledge. We evaluate ELK with 76 pre-service teachers in two modes, one is more akin to the kind of human-human interaction that is germane to teaching but requires more human resources and one that can be done independently. We find that in both cases ELK raises awareness about student misconceptions and the importance of student thinking, and teachers demonstrate a modest increase in effective questioning strategies after three rounds of playing.
\end{abstract}

\section{Author Keywords}

Professional training; teacher education; simulation; role-playing game; focused practice.

\section{CCS Concepts}

-Applied computing $\rightarrow$ Computer-assisted instruction; Interactive learning environments;

\section{INTRODUCTION}

With increasing challenges associated with the rapidly changing landscape of work, technology-based solutions that support professionals in lifelong learning are in more demand. In particular, teaching, like many complex skills, poses challenges for exercising relevant skills genuinely, and thus, many professional training opportunities for teachers fail to offer such authentic practice. In this work, we develop and evaluate a role-playing game that helps teachers learn and practice one important classroom discourse strategy: eliciting learner knowledge. ELK is a two-person, chat-based simulation where pre-service teachers can practice question asking strategies and role-play as students with developing understanding of STEM concepts. With the goal of achieving maximum impact with the most scalable and convenient solution, we evaluate two modes in ELK and we demonstrate how both of these early prototypes of our system cultivate empathy with students and lead to improvements in questioning strategies.

Effective teacher learning and professional development is important for student achievement. Teacher learning is a continuous process that promotes teachers' teaching skills, supports them in developing new proficiency, which in turn, helps improve students' learning. In the United States, teacher professional learning programs provide insufficient opportunities for teachers to practice important skills and judgments [12]. "Pre-service" teacher training - the process of initial licensure in colleges of education - typically combines classroom-based course work with field observations and practicum teaching [17]. In-service teacher training programs take the form of professional seminars, workshops, expert or peer classroom observation and consultations [5, 13, 25]. For both pre- and in-service teachers, teacher learning consists primarily of lecture or discussion with few opportunities to rehearse teaching practice and receive meaningful feedback. Teachers are expected to gradually pick up skills "on the job" as they teach in real classrooms, in a haphazard process of trial and error with insufficient feedback. We align our work with a movement of teacher-educators advancing practice-based teacher education, an approach to teacher professional learning that emphasizes opportunities for rehearsal and reflection [28].

Despite decades of research showing the benefits of studentcentered teaching practices, the shift from teacher-centered practices has been slow [11]. Although research and practice has shown that in teacher, understanding what students know is essential to helping them learn [6]; Attending to students' thinking allows teachers to discover student misconceptions [21] and tailor instruction [1]. Pre-service teachers often regard students' misconceptions as barriers to learning, rather than useful starting points for instruction [16]. Even seasoned teachers often focus on evaluating student work as right or wrong, rather than understanding student work as evidence of current understandings that can be built upon [7]. We aim to 
improve the situation by designing a role-playing game to help educators both recognize the importance of eliciting learner knowledge and to learn and practice strategies to understand what students know.

We present ELK (Eliciting Learner Knowledge), a game that offers online virtual role-playing sessions in which teacher candidates can learn and practice discourses strategies on eliciting learner knowledge. The systems offers a scalable and practice-based solution for teacher professional training around classroom discourse. As a digital system, the platform archives teacher conversations that supports both teacher reflection and review of practice and allows researchers to investigate the processes of teacher learning in granular detail.

ELK provides two modes of play. In the "Chat" mode, two players each assume the role of a "teacher" or a "student" with pre-written profiles. The "student" profile presents a realistic set of conceptions and misconceptions about a topic (such as the causes of seasons in the northern hemisphere). The "teacher" profile sets out questioning goals for the teacher player ("does the student understand the role of the Earth's tilt in seasonality?") The goal of the "teacher" player is to elicit prior knowledge of the "student" player as scripted. The two players type to chat. Each session lasts 7 minutes. In the "Code" mode, players play individually. Players come in and evaluate past users' conversation transcripts collected on ELK. Players need to assign a qualitative code for to each line of the transcript, indicating which questioning move was employed such as "Telling", "Evaluating", or "Probing."

We evaluate ELK with 76 pre-service teachers in two modes. In the remainder of the paper, we refer to pre-service teachers as PST. The "Chat" mode is more akin to the kind of human-human interaction that is germane to teaching but requires more human resources and the "Code" mode can be done more independently thus offering a convenience benefit. We find that in both cases ELK raises awareness about student misconceptions and the importance of student thinking, and teachers demonstrate a modest increase in effective questioning moves in eliciting learner knowledge after three rounds of playing, with no penalty associated with the increased convenience of the more independent version. Our work demonstrates promise in using simulations to give teachers more practice for skill development and support teachers in developing sympathy with students through role-playing. Our work also shows that it might be valuable for teacher training platforms to offer a variety of activities. For example, structured and more scalable activities, such as evaluating past transcripts, can be beneficial for novice teachers to develop conceptual understanding of what are positive and negative practices.

\section{DESIGN MOTIVATIONS OF ELK}

The design of ELK is motivated by a review of literature related to what is missing from current teacher training practice. The game design takes ideas from existing frameworks on how to teach teachers to elicit learner knowledge and learning science theories that suggest giving novices (in this scenario, pre-service teachers) focused practice and appropriate scaffolding is important for skill development.
Simulations can Provide Meaningful Training Experiences Many other professions equip practitioners with extensive hands-on training and practice opportunity before they start their professional life, such as nurses $[3,19]$. However, teachers often do not get sufficient opportunities to practice teaching in low stakes settings $[12,17]$. When teachers do engage in low-stakes simulated practice, it is often in the form of "rehearsals" where participants practice teaching an entire activity or lesson to a group of simulated students [15]. Teaching is immensely complex, and research on complex learning suggests that novices often struggle to practice a whole complex assemblage while improving at specific elements of the task [14]. Rehearsals of the whole assemblage of teaching, therefore, should be complemented by opportunities to practice more discrete skills and judgments in teaching practice [20].

Teacher education researchers have explored a variety of approaches to improving discrete elements of practice such as questioning strategies. One method involves recording teachers' one-on-one conversations with students and have experts review them afterwards [24]. Another approach involves creating face to face simulations, where teacher educators act a students with misconceptions, and pre-service teachers practice questioning strategies. [22]. These are compelling approaches, but they are very demanding on teacher educator labor (to review video, act as students, provide individual feedback, etc.) We build upon these promising approaches by creating a digital platform for practicing question asking strategies in a peer-learning context, where teacher education students play both roles of teachers and students and provide each other feedback on discrete elements of practice: eliciting learner knowledge.

\section{How to Elicit Learner Knowledge}

Prior work has studied effective "talk moves" for teachers to elicit learner knowledge, such as asking follow-up questions $[6,8,10,18]$. We reviewed prior work and then developed a framework for training pre-service teachers on effective questioning moves. The framework contains five categories of questioning moves.

- Priming: preparing the class for learning

- Eliciting: asking questions that reveal learner's needs

- Probing: asking follow-up questions based on students' responses

- Evaluating: responding in the positive or negative about students' answers

- Telling: talking about the topic without listening to the student

Among the five questioning moves, we consider Priming, Eliciting and Probing to be effective ones in eliciting learner knowledge; and Evaluating and Telling to be ineffective ones for eliciting knowledge, since they are not optimal for teachers to understand what students know. (Telling and evaluating may be appropriate in other parts of the teaching sequence, but in the early phases of eliciting learner knowledge, it is critical to simply understand student thinking before attempting to "fix" or redirect student thinking. 


\section{Algebra Grade 6}

You are a 6th grade student in math class. Your teacher is about to start a lesson on variables and equations, and would like to see what you already know.

\section{Your Student Profile:}

Any letter given to me has the value of the where that letter is in the alphabet. A (or a) always has a value of $1, b$ always has a value of 2 , and so on. Even if the problem tries to trick me by saying $a=3$, I will always input the value of 1 for a. I will always convert to numbers before I do anything else with the problem. If anything is given to me without operation symbols $\left(+,{ }^{*},-, /\right)$, I will just stick the number in front of that value. An example would be 3a. I know that a has to be 1. The value of this would be 31.
When you are ready to begin the round, click Begin

\section{Begin}

Use the scenario to the left to guide your conversation:

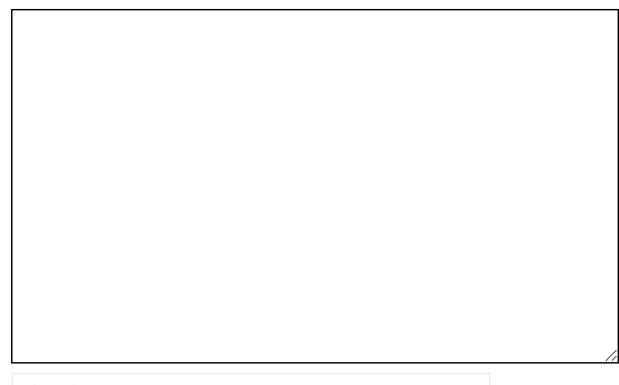

Chat here

Send Message

When the 7 minute round is finished, take the quiz. Take Quiz

Figure 1. "Chat" mode of ELK: student profile showing (mis)conceptions on the left, and the chatting interface on the right where players will have "teacher-student" conversations.

This five-part questioning framework is central to our pedagogical aims and research efforts with ELK. In implementing ELK in educational methods classes, teacher educators explain and demonstrate these questioning strategies to pre-service students. ELK participants then use these questioning strategies to code talk moves in transcripts. In analyzing ELK transcript data, as researchers we use this framework to identify preservice teacher development - we consider an increase in the three effective questioning strategies and the decrease in the two ineffective strategies over multiple rounds of ELK to be evidence of pre-service teacher fluency in eliciting learner knowledge.

\section{Practice Through Solution Generation Or Evaluation}

The ELK platform provides two pathways, grounded in cognitive science and instructional design, for pre-service teachers to develop effective questioning strategies. An open question in instructional design involves what practice gives learners (PSTs) the most learning benefit. Consider alternative ways of practice, "solution generation" and "solution evaluation." With our instructional goal of teaching PSTs effective questioning moves, "solution generation" would involve practices of generating questions to elicit learner knowledge, and "solution evaluation" may involve practices of evaluating the quality of question moves made by themselves or others.

Some theories suggest that the constructive or generative processes evoked by "solution generation" produce more learning than the active but non-generative processes evoked by "solution evaluation" [4, 23]. Deliberate practice [9] provides an alternative argument that what is most important to learning is whether the instructional activity "solution generation" or "solution evaluation" involves elements of the desired competence that are at the edge of learners' capability $[2,9]$. Prior work has shown that in some domains, evaluating the quality of solutions can support learning and performance on generating solutions afterwards, even with higher learning efficiency compared with practicing with generating solutions only. For example, Yannier et al. shows that evaluating "which towers would likely to fall" can be more effective in teaching kids physics principles around gravity and balance compared to having kids continuously build towers with LEGO [27]. Wang et al. shows that evaluating candidate solutions can be equally effective in teaching college students how to design good survey questions compared to having students practice through generating survey questions [26].

We see a potential contrast in "solution generation" and "solution evaluation" in the domain we are focusing on. When PSTs are learning effective questioning moves, does the challenge lie in coming up with new questions or evaluating which question moves are more effective in eliciting learner knowledge? With this in mind, we implemented two modes of play in ELK, each highlighting the "solution generation" process - "Chat" mode, and the other highlighting the "solution evaluation" process - "Code" mode. On a separate note, the "Code" mode can be more convenient and scalable compared to the "Chat" mode, which doesn't require a partner to be present. The comparison between the two modes contributes to the instructional design literature and at the same time helps inform interaction designs of future professional training simulations. 


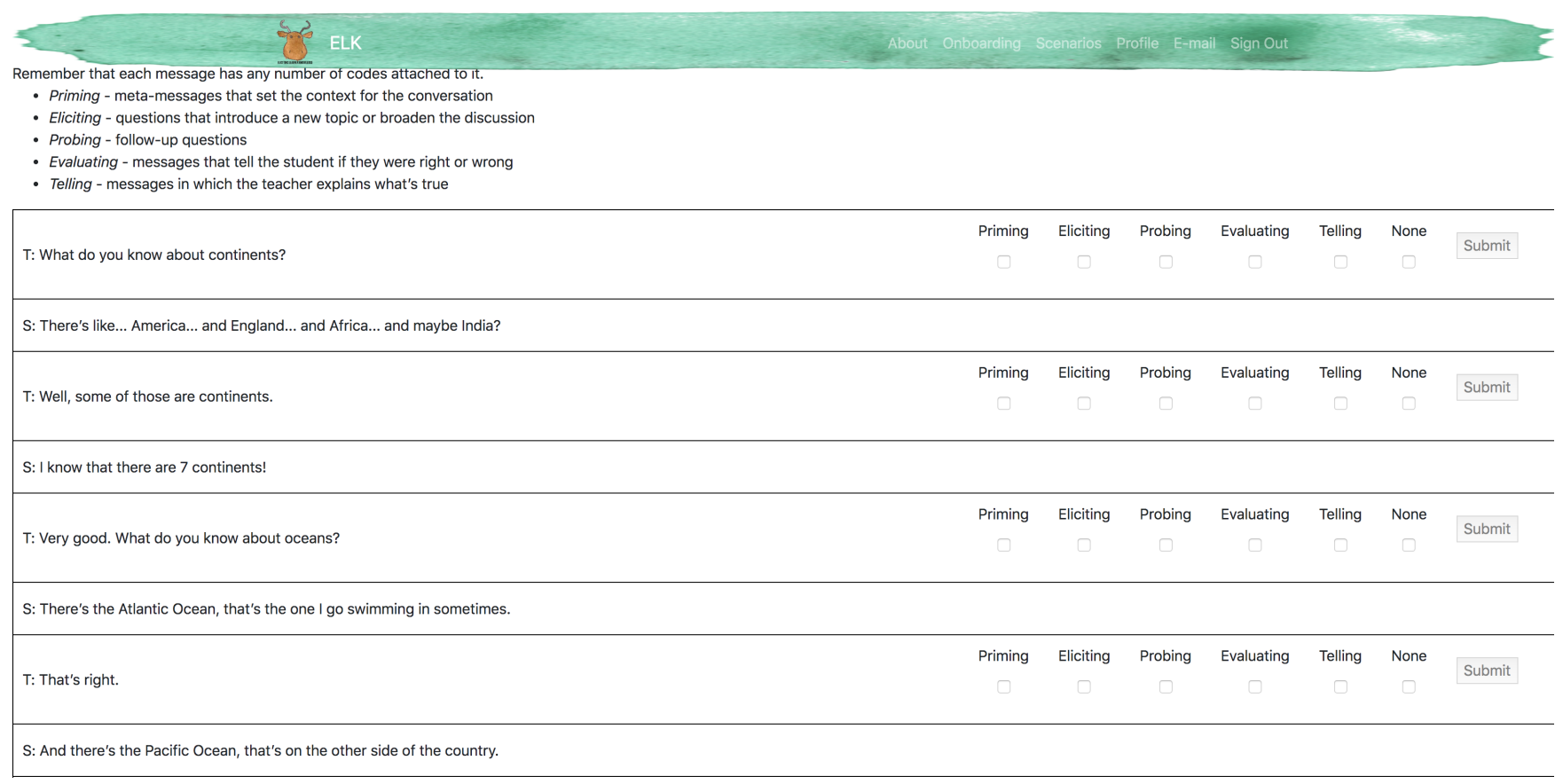

Figure 2. "Code" mode of ELK: evaluating a past "teacher-student" conversation transcript.

\section{ELK: A ROLE-PLAYING SIMULATION GAME}

The design of ELK aligns with the core principles described above: we developed a simulation platform that supports a practice-based approach to developing a key teaching skill: eliciting learner knowledge through practice and rehearsal. Our system lets participants develop questioning strategies through generating new questions (improvisationally, in a realtime conversation) and through evaluating prior participants questions. We use the transcript data generated by rounds of ELK game play to evaluate participant learning over time, and ideally over multiple rounds of ELK, participants would demonstrate increased proficiency through the increased use of effective questioning strategies, reduced use of ineffective (but common strategies), and overall improvement in eliciting key features of student thinking on STEM topics.

In the "Chat" mode that offers focused practice on "solution generation", two players each take the role of a "student" or a "teacher" and have one-to-one conversations in a chat interface. After entering the platform, players first select a topic, e.g., grade 6 algebra, or grade 3 multiplication. The "student" role player enters the interface as shown in Figure 1 with a "student" profile. The "student" profile specifies prior knowledge held by the player, and the player should play out this persona. The "teacher" role player enters the same interface with a "teacher" profile, which specifies the objectives of the "teacher", e.g., "Solve real-world and mathematical problems by writing and solving equations of the form $\mathrm{x}+\mathrm{p}=\mathrm{q}$ " on the topic of grade 6 algebra. In each chat session, the goal of the "teacher" is to elicit the prior knowledge of the "student" player, including both accurate knowledge and misconceptions. The shown example here is on the topic of grade 6 algebra. On the ELK platform, players can pick from a list of topics with profiles written by the developers of the game, or teacher educators or PSTs can develop new profiles.

In the "Code" mode that offers focused practice on "solution evaluation", players participate in a qualitative coding activity. Players evaluate "teacher-student" conversation transcripts by past players of ELK and decide which questioning move the "teacher" was using in the chat, as shown in Figure 2. Players are expected to understand what are the desirable and undesirables questioning moves with concrete examples. Compared to traditional training methods, such as giving PSTs reading materials and guidelines, ELK "Code" mode supports active learning [4], which has been found to be better than passive learning. ELK is publicly available at: https://newelk. herokuapp. com $^{1}$

\section{EVALUATION OF ELK}

We evaluate ELK in an authentic teacher training context. The experiment was conducted at a small liberal arts college in the Mid-Atlantic region. 80 undergraduate students (PSTs) from a teacher training program participated in the experiment. We ask two research questions in this experiment. First, is ELK helping pre-service teachers learn effective questioning moves? Second, is the combination of "Chat" and "Code" play more helpful for learning than "Chat" only? An alternative way of framing the second question is, with learning effective effective questioning moves as the goal, is practice through "solution generation plus evaluation" more helpful for learning than practice through "solution generation" only?

\footnotetext{
${ }^{1}$ Reviewers are welcome to use these test accounts to try out the game. Pick one of the five usernames: [CHIGuest1, CHIGuest2, CHIGuest3, CHIGuest4, CHIGuest5], the password is the same for all accounts: ELKELKELK. Please note that for the "Chat" mode,
} 


\section{Experiment Design}

The experiment was incorporated into the curriculum of four classes on teacher education. The experiment happened during two 90-minute class meetings. Three instructors were involved and they followed the same procedure as shown in Figure 3. In the first class meeting, the instructor first gave a 15-min lecture on effective questioning moves, with definitions and examples of each of the five questioning moves we introduced earlier. All participants were randomly divided into two conditions. In each condition, participants were randomly assigned into pairs. Each pair played ELK together. Between the pair, the instructor randomly assigned one to play the "teacher" role, and the other to play the "student" role. All participants consistently played either the "teacher" or the "student" role across three rounds. The only difference between conditions happens in Round 2, where one group did the "Chat" mode, and the other did the "Code" mode. This design allows us to see whether participants' performance in the chats become better from Round 1 to Round 3 if they received different treatments in Round 2.

In the second class meeting, all participants get to switch roles and experience the "Code" mode if they have not done so. By the end of the second class meeting, we made sure all participants had experienced both "teacher" and "student" roles, and both modes of the game. We then sent a post-survey to ask about participants' experience and feedback in the game.

two players need to be present. For the "Code" mode, please go to Onboarding and select "Learning to ELK as a teacher."

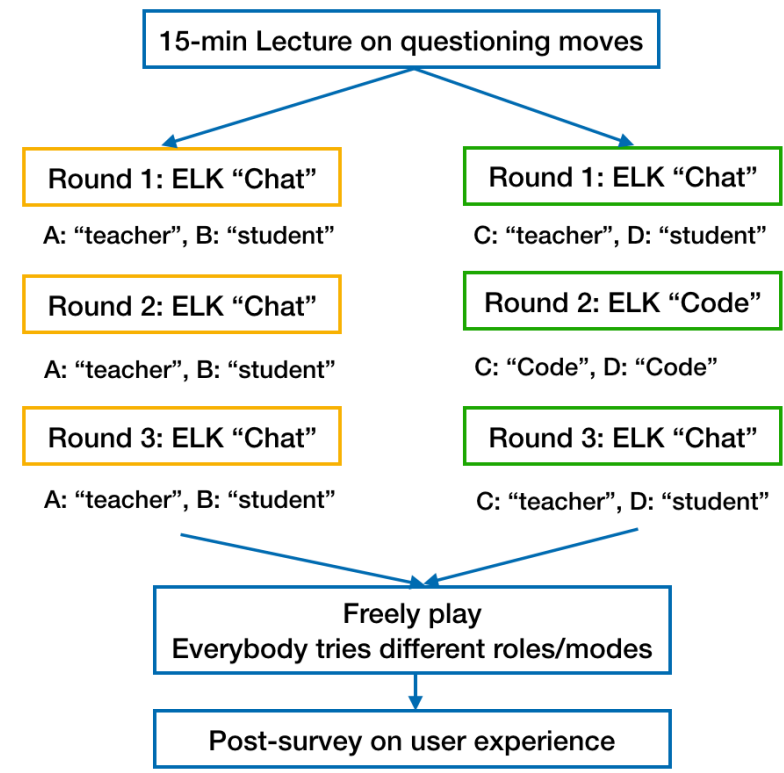

Figure 3. Experiment procedure across two 90-minute class meetings. In the first class meeting, participants play three rounds of ELK. All students play one Chat round, and then students are randomly assigned to play either "Code" or "Chat" mode in Round 2. In the second class meeting, all participants get to play both roles and try both modes. They then ask a user experience survey in the end.

\section{Learning Outcome Measures}

The goal of this game is to help PSTs become more effective in eliciting learner knowledge. We used PSTs performance in the chats as pre-test and post-test measures. For PSTs who played the "teacher" role, we analyzed the questioning moves they employed in Round 1 and Round 3 of playing ELK. For PSTs who played the "student" role, we do not have a learning measurement.

More specifically, we developed a coding manual to assess PSTs' questioning moves in the chats. The coding manual was developed over an iterative process. In the coding manual, for each category of questioning move, we provide multiple examples including explanation of edge cases. The coding manual is available in supplemental material. A brief version of the coding manual is shown in Table 1.

When the goal is to figure out what the student already knew, questioning moves of "Priming", "Eliciting" and "Probing" are considered to be positive moves, and "Telling" and "Evaluating" are considered to be negative moves in this context, because they are not supporting the teacher to understand student (mis)conceptions and are not helpful for facilitating student-centered instruction.

Two researchers independently coded a subset of data and reached high agreement on the 5 questioning moves in the coding manual, with Cohen's Kappa of 0.73 for Priming, 0.8 for Eliciting, 0.72 for Probing, 0.76 for Evaluating and 0.78 for Telling. In coding practice, we assign questioning move codes for "teacher" turn. The 5 codes are not mutually exclusive, which means a "teacher" turn can be both "Priming" and "Eliciting." We then sum up the number of effective moves ("Priming", "Eliciting", "Probing") and the number of ineffective moves ("Telling", "Evaluating") in a chat for each PST as their pre-test and post-test score.

\section{EXPERIMENT RESULTS}

\section{Definition of Variables}

Among the 80 PSTs participated, 4 did not complete the activities as required, thus were excluded from the analysis. Among the rest of PSTs, 38 played the "teacher" role and had a learning measure. The dataset only contains the PSTs who played the "teacher" role. In the dataset, each row represents one chat(observation) of a player. Each row contains the following variables, which are standardized in the analysis.

- PositiveMoves: This is a continuous numeric variable that measures the number of effective questioning moves in a chat.

- NegativeMoves: This is a continuous numeric variable that measures the number of ineffective questioning moves in a chat.

- Condition: This is a binary variable, being either "Chat" or "Code." This indicate during the second round of activity, which condition the PSTs engaged in.

- Pre/post-test: This is a binary variable, indicating whether the chat is "pre-test" or "post-test." To clarify, "pre-test" here refers to the "teacher" performance in Round 1, "posttest" refers to the "teacher" performance in Round 3. 


\begin{tabular}{|c|c|c|}
\hline $\begin{array}{l}\text { Questioning } \\
\text { Move }\end{array}$ & Definition & Example \\
\hline \begin{tabular}{l|l} 
Priming \\
\end{tabular} & $\begin{array}{l}\text { These are meta-messages that set the context for the conversa- } \\
\text { tion. They might appear at the beginning of the conversation } \\
\text { or later to bring the conversation back to the topic/goal. }\end{array}$ & $\begin{array}{l}\text { [after a student asks, "Is that right?"] I will tell you all about } \\
\text { that during class today, but for now I just want to understand } \\
\text { your ideas. }\end{array}$ \\
\hline Eliciting & $\begin{array}{l}\text { These are questions that introduce a new topic or broaden } \\
\text { the discussion. }\end{array}$ & $\begin{array}{l}\text { Teacher: If I were to give you } x+p=q \text {, would you know how } \\
\text { to solve? } \\
\text { What do you know about the area of a circle? }\end{array}$ \\
\hline Probing & $\begin{array}{l}\text { These are follow-up questions that go deeper into what the } \\
\text { student thinks. It is often impossible to tell the difference } \\
\text { between Eliciting and Probing messages without context. }\end{array}$ & $\begin{array}{l}\text { Teacher: What do you know about word order? (Eliciting) } \\
\text { Student: The noun comes before the verb. } \\
\text { Teacher: Is this always true? Or are there ever nouns after } \\
\text { verbs in a sentence? (Probing) }\end{array}$ \\
\hline Evaluating & $\begin{array}{l}\text { These messages tell the student if they were right or wrong, } \\
\text { either explicitly or implicitly. They often lead to Telling } \\
\text { messages and distract the student from the goal of figuring } \\
\text { out their preconceptions. }\end{array}$ & $\begin{array}{l}\text { That's right. } \\
\text { Not quite. }\end{array}$ \\
\hline Telling & $\begin{array}{l}\text { These are messages in which the teacher explains what's } \\
\text { true. While important during instruction, they are distracting } \\
\text { if the goal is to figure out what the student already knew or } \\
\text { believed. }\end{array}$ & The circumference of a circle in $2 \pi r$. \\
\hline
\end{tabular}

Table 1. Brief version of the coding manual, with definitions and examples of the 5 questioning moves.

- WordsPerChat: This is a continuous numeric variable that measures the total number of words said by the "teacher" player in an entire chat transcript.

\section{Teachers Displayed More Effective Questioning Moves}

We first ran a paired t-test from pre- to post-test on PositiveMoves. We find that PSTs displayed significantly more positive moves in post-test than in pre-test $(p=0.014)$. The average number of positive moves per chat in pre-test was 4.5 , and the average number in post-test was 5.1. There is no significant difference between pre- to post-test on NegativeMoves.

\section{A Combination of Solution Generation And Evaluation is \\ Better}

All students practiced generating questions in Rounds 1 and 3 , and half of participants generated questions in Round 2 while the other random half evaluated questions using the "Code" mode in ELK. To evaluate whether the combination of question generation and evaluation supports learning better than question generation alone, we evaluated the Round 3 counts of positive and negative questions types between the two treatment groups.

In a simple linear regression model with PositiveMoves as the dependent variable, Condition, Pre/post-test, and their interaction term as factors, and controlled for WordsPerChat. The parameters of the model is shown in Table 2. The average

\begin{tabular}{l|l|l}
\hline Variables & Coeff & $\boldsymbol{p}$-value \\
\hline Treatment (Chat) & 0.057 & 0.219 \\
\hline Pre/post-test (Post-test) & 0.040 & 0.213 \\
\hline Words per chat & 0.614 & 0.000 \\
\hline Treatment * Pre/post-test (Chat * Post-test) & -0.100 & 0.29 \\
\hline
\end{tabular}

Table 2. Model 1: Regression model results on the full dataset. number of positive moves in pre- and post-tests for both conditions is shown in Figure 4. The result suggests that "Code" condition is significantly better than "Chat."

However, in evaluating Round 1 measures of positive and negative questioning, we observed that our randomization produced unbalanced groups: the Round 1 counts of positive questioning moves in the "Code" condition are significantly lower than the counts of positive moves in the "Chat" condition. the larger improvement when players did a combination of "Chat" and "Code" could be because they had lower baseline scores and more room for improvement.

To address this, we did propensity score matching to match players that had similar performance in pre-test but were in different conditions. 20 participants were matched. In this matched subset, we did the same linear regression model again, the output of the model is shown in Table 3. The average number of positive moves in pre- and post-tests for both conditions is shown in Figure 5. Consistently, we are seeing a trend that the increase for the "Code" condition is higher than the "Chat"

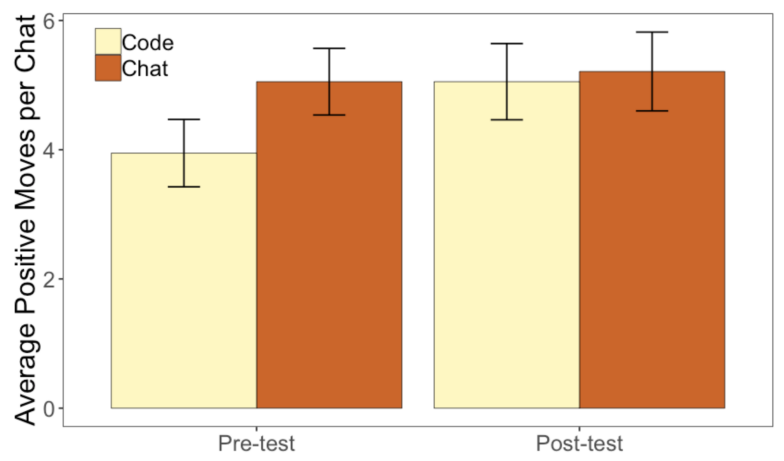

Figure 4. Average positive behaviors from first to last chat across both conditions for the full dataset. 


\begin{tabular}{l|l|l}
\hline Variables & Coeff & $\boldsymbol{p}$-value \\
\hline Treatment (Chat) & -0.044 & 0.408 \\
\hline Pre/post-test (Post-test) & 0.100 & 0.027 \\
\hline Words per chat & 0.647 & 0.000 \\
\hline Treatment * Pre/post-test (Chat * Post-test) & -0.100 & 0.103 \\
\hline
\end{tabular}

Table 3. Model 2: Regression model results on the subset data, with players matched using propensity score matching.

condition. The difference in increase is marginally significant $(p=0.103)$, and substantively modest.

\section{Chat Examples}

Here is one example of behavior change of participant P68 (Figure 6). In pre-test, the PST used "Evaluating" and "Telling" questioning moves. In post-test, in a similar situation, the PST started to use "Eliciting" and "Probing."

\section{Discussion}

From the experiment, we see a modest increase in effective questioning moves for both conditions from Round 1 to Round 3 , and the increase for the condition who did a combination of "Code" and "Chat" practice is slightly higher. This addresses our research question that in terms of learning to ask good questions, getting practice through evaluating solutions can be beneficial. The increase in effective questioning moves is smaller than what we would have expected. We did not observe a change in negative moves between Round 1 and Round 3. It could be because that the dosage of intervention we used in the experiment is not enough for significant behavior change. Future work should consider using large dosage of interventions and longitudinal studies to make conclusions about the effects.

\section{PLAYER EXPERIENCE WITH ELK}

Following the second class meeting where everybody gets to experience both roles and modes, we sent an experience survey to all participants to gather feedback on different aspects of the ELK platform to inform future design of ELK and relevant teacher training programs. The survey includes three types of questions, 1) asking whether and how ELK helped with learning of questioning moves, 2) asking whether and how ELK helped PSTs understand what students know, 3) whether participants had any suggestions for future improvement.

\section{Experience and Feedback}

We took a grounded theory approach to analyze participants' responses in the survey. We first coded the participants' openended response and marked useful concepts and key phrases, and we combined common categories as they appear. we finally summarized the data into several themes as follows.

\section{Getting Practice is Helpful}

Participants mentioned that getting practice on eliciting learner knowledge of ELK is helpful.

"It helped to give some practice in phrasing questions so as to get the most helpful info from students in the most direct way possible." - P68

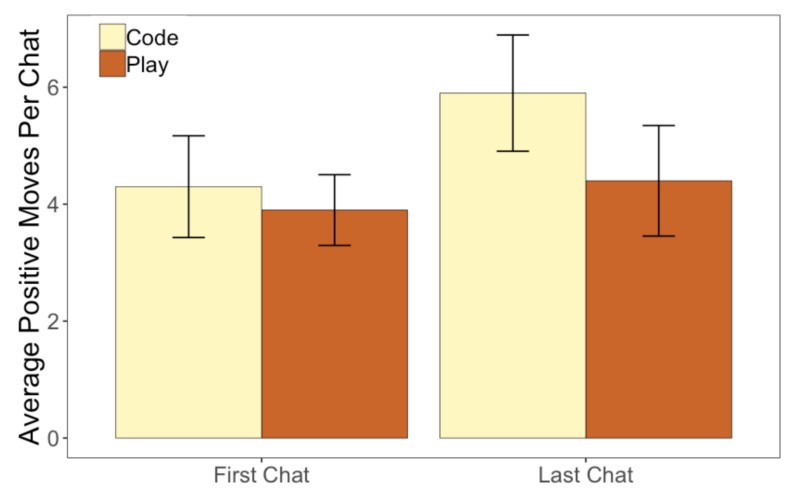

Figure 5. Average positive behaviors from first to last chat across both conditions on the subset data, with players matched using propensity score matching.

"ELK gave us a space to practice using different types of questions and see how to best retrieve knowledge from a student." - P5

\section{Changed My Behavior in the Game}

Some participants talked about learning question moves from the game and changed the way they asked questions.

"I made a few changes to the way I asked questions and I ended up asking more questions rather than trying to explain the material." - P32

"Role-playing as a teacher and understanding the different types of questions was effective in a sense that I now know how to understand students' prior knowledge on a topic without blatantly telling them." - P21

"ELK helped me learn how to ask particular questions to get students to reconsider their answers and think of new ideas. Directly telling a student if they are right or wrong is ineffective and should be used sparingly. Teachers should guide students to new knowledge by asking questions that prompt their thinking." - P55

\section{Coding is Helpful}

Some participants said that the coding activity was helpful for them to know the difference between questioning moves and some suggested to include coding activities at an earlier stage in the platform.

"It helped me to really know the difference between eliciting and probing and how to ask good, open-ended probing questions." - P30

"ELK helped me learn questioning strategies because it allowed me to code information and questions and use that information to ask questions during the teacher role." - P46

"Maybe include more with the coding section." - P64

"I found the coding very helpful. would be more helpful if done at very beginning instead of midway through." P27 

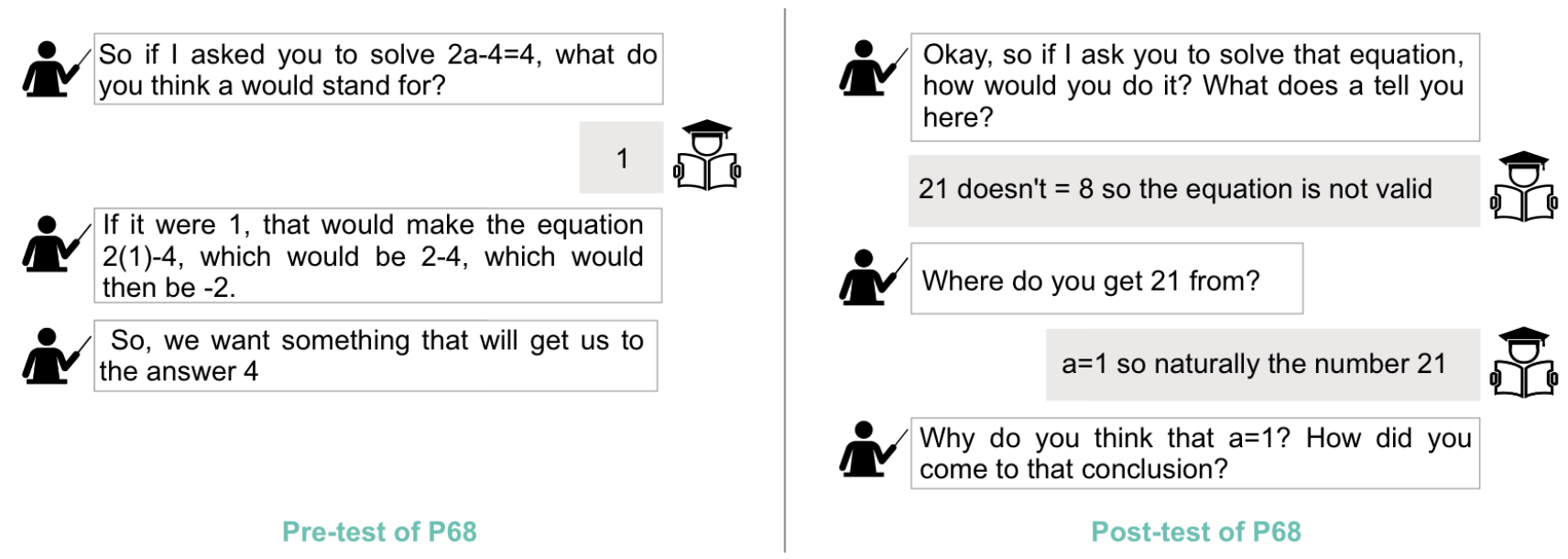

Figure 6. Left: excerpts of pre-test of P68: the teacher used "Evaluating" and "Telling" moves when the student gives an unexpected answer. Right: excerpts of post-test of P68: When the student gives unexpected answer, the teacher used "Eliciting" and "Probing" moves to understand the thinking process of the student.

\section{Helps Develop Sympathy With Students}

The most frequent theme came out from participant responses was that ELK helped them be aware the different perspectives and conceptions students might have, especially through playing the "student" role. Some participants found it surprising that students can think in such different ways.

"it was just eye opening to see how different students think and how you can respond to them." - P2

"When learning about variables, students thought the alphabet and the certain letter was paired up with where the number is in the alphabet. I never thought about it like that until then." - P11

Some participants summarized the takeaway as since students might think very differently from teachers and might give surprising responses to questions, teachers need to be patient, open-minded, and flexible in teaching and need to learn to adjust to different situations. We consider these as important takeaways from the game.

"Students can come up with some odd associations, and seeing some of them written out helped remind me to be more flexible as a teacher because sometimes the associations make sense to others as well, and sometimes they only barely make sense to the student, so the teacher needs to be patient and open-minded." - P68

"It shows that all students have different knowledge and different ways of thinking, and teachers must adapt quickly to answer questions that their students have." P58

"ELK helped me by showing me that sometimes you may have to ask off the wall questions to even see if the student has any misconceptions because they could be thinking something that is completely wrong and you think it is common sense to know but they do not know and we as teachers need to get to that point and help them to understand the content area we are teaching at the time." $-P 24$

\section{DESIGN RECOMMENDATIONS}

From the quantitative evaluation, qualitative analysis of survey responses, we summarized three design recommendations to be considered for teacher training programs.

\section{Focused Practice on Generation And Evaluation}

The experiment suggests that having PSTs evaluate past transcripts can be an effective instructional activity. In future implementations, we plan to test different sequences of question generation and evaluation, perhaps starting with more evaluation activities early on - to rapidly develop familiarity with question-types in an authentic discussion, before moving to generation.

\section{Sympathy With Students}

One important finding from the user experience survey is that a large proportion of the participants expressed that ELK helped them raise awareness about the misconceptions students may hold and the variety of thinking processes that students may have. This suggests that perspective taking is important. Preservice teachers may seldom have the experience to look at things from students' eyes. This also points to the potential of role-playing simulations for professional training for teachers or other helping professions to enable trainees take a different perspective and develop sympathy with other stakeholders in the process.

\section{Content Domain Knowledge is Essential}

When asked about the challenges encountered while playing the game, many participants mentioned that they do not have enough domain knowledge of the topic given, thus were not able to ask questions effectively.

"It helped me learn how to ask questions without giving too much feedback. The content confused me greatly with the heredity though, I did not know what was right or wrong and was really confused. I don't remember much about it since I have not taken a Bio class in 4 years." P26 


\begin{abstract}
"Giving us a little bit of background knowledge on the topics of the lessons before hand so we actually know what we are teaching to the students." - P50
\end{abstract}

We can see this confusion might happen in many teacher training contexts. Pre-service teachers are being trained on teaching skills. However, teacher training requires the trainees to have basic domain content knowledge. In the ELK platform, in addition to choosing one of the given topics, players can write profiles themselves and upload to the platform. We consider that to be a possible feature to address this. Most teacher training programs use static materials which might confuse teachers who are not experts on the content. Allowing pre-service teachers to create and use content that they're familiar with could be helpful for them to learn new teaching skills. Additionally, if the content can be shared, over time, new trainees can just pick topics from a pool instead of writing new ones.

\section{CONCLUSION}

All across the helping professions - teaching, medicine, social work, clerical work, and so forth - eliciting thoughts, feelings, and understandings from people is a critical part of professional practice. In this work, we demonstrate a system that supports simulated practice of questioning strategies through two learning modalities: question generation and question evaluation. In a teaching context, we find evidence that ELK helps participants value learner knowledge, empathize with the challenges of students as they develop understanding of STEM topics, and increase their use of questioning strategies that effectively elicit learner knowledge. We have tentative evidence that a combination of question generation and evaluation practice may be more effective than question generation practice alone in increasing the use of teacher questioning strategies.

While the scenarios in ELK are customized primarily to deal with STEM topics; ELK could be customized to support the development of interpersonal skills in a wide variety of contexts and professions. ELK's digital platform supports a variety of educational implementations (in face to face classes, in online classes, as out of classwork, etc.) and collects data for participants, educators, and researchers to better understand how learners develop effective questioning strategies.

\section{REFERENCES}

[1] Susan A Ambrose, Michael W Bridges, Michele DiPietro, Marsha C Lovett, and Marie K Norman. 2010a. How learning works: Seven research-based principles for smart teaching. John Wiley \& Sons.

[2] Susan A Ambrose, Michael W Bridges, Michele DiPietro, Marsha C Lovett, and Marie K Norman. 2010b. How learning works: Seven research-based principles for smart teaching. John Wiley \& Sons.

[3] Ewa Pihammar Andersson. 1993. The perspective of student nurses and theirperceptions of professional nursing duringthe nurse training progranune. Journal of Advanced Nursing 18 (1993), 808-815.
[4] Michelene TH Chi and Ruth Wylie. 2014. The ICAP framework: Linking cognitive engagement to active learning outcomes. Educational Psychologist 49, 4 (2014), 219-243.

[5] Nancy Van Note Chism, Matthew Holley, and Cameron J Harris. 2012. 9: RESEARCHING THE IMPACT OF EDUCATIONAL DEVELOPMENT: BASIS FOR INFORMED PRACTICE. To improve the academy 31, 1 (2012), 129-145.

[6] National Research Council and others. 2004. How students learn: History, mathematics, and science in the classroom. National Academies Press.

[7] Sandra Crespo. 2000. Seeing more than right and wrong answers: Prospective teachers' interpretations of students' mathematical work. Journal of Mathematics Teacher Education 3, 2 (2000), 155-181.

[8] Brent Duckor and Carrie Holmberg. 2017. Mastering formative assessment moves: 7 high-leverage practices to advance student learning. ASCD.

[9] K Anders Ericsson, Ralf T Krampe, and Clemens Tesch-Römer. 1993. The role of deliberate practice in the acquisition of expert performance. Psychological review 100, 3 (1993), 363.

[10] ERIN MARIE FURTAK and HOWARD M GLASSER. 2016. Using Formative Assessment Data for Science Teaching and Learning. (2016).

[11] David Gerritsen. 2018. A socio-technical approach to feedback and instructional development for teaching assistants. Ph.D. Dissertation. Carnegie Mellon University.

[12] Pamela Grossman, Christa Compton, Danielle Igra, Matthew Ronfeldt, Emily Shahan, and Peter Williamson. 2009. Teaching practice: A cross-professional perspective. Teachers College Record 111, 9 (2009), 2055-2100.

[13] Andy Jacob and Kate McGovern. 2015. The Mirage: Confronting the Hard Truth about Our Quest for Teacher Development. TNTP (2015).

[14] Paul A Kirschner and Jeroen Van Merriënboer. 2008. Ten steps to complex learning a new approach to instruction and instructional design.

[15] Magdalene Lampert, Megan Loef Franke, Elham Kazemi, Hala Ghousseini, Angela Chan Turrou, Heather Beasley, Adrian Cunard, and Kathleen Crowe. 2013. Keeping it complex: Using rehearsals to support novice teacher learning of ambitious teaching. Journal of teacher education 64, 3 (2013), 226-243.

[16] Douglas Larkin. 2012. Misconceptions about "misconceptions": Preservice secondary science teachers' views on the value and role of student ideas. Science Education 96, 5 (2012), 927-959.

[17] Arthur Levine. 2006. Educating school teachers. Education Schools Project (2006). 
[18] Sarah Michaels and Catherine O'Connor. 2015. Conceptualizing talk moves as tools: Professional development approaches for academically productive discussion. Socializing intelligence through talk and dialogue (2015), 347-362.

[19] Sonia Moore. 1984. The Stanislavski system: Professional Traning of an Actor. Viking Penguin Inc.

[20] Justin Reich, Yoon Jeon Kim, Kevin Robinson, Dan Roy, and Meredith Thompson. 2018. Exploring Authenticity and Playfulness in Teacher Practice Spaces. (2018).

[21] Philip M Sadler, Gerhard Sonnert, Harold P Coyle, Nancy Cook-Smith, and Jaimie L Miller. 2013. The influence of teachers' knowledge on student learning in middle school physical science classrooms. American Educational Research Journal 50, 5 (2013), 1020-1049.

[22] Meghan Shaughnessy and Timothy A Boerst. 2018. Uncovering the skills that preservice teachers bring to teacher education: The practice of eliciting a student's thinking. Journal of Teacher Education 69, 1 (2018), 40-55.

[23] Norman J Slamecka and Peter Graf. 1978. The generation effect: Delineation of a phenomenon. Journal of experimental Psychology: Human learning and Memory 4, 6 (1978), 592.
[24] Laurie Sleep. 2012. The work of steering instruction toward the mathematical point: A decomposition of teaching practice. American Educational Research Journal 49, 5 (2012), 935-970.

[25] Ann Stes, Liesje Coertjens, and Peter Van Petegem. 2010. Instructional development for teachers in higher education: Impact on teaching approach. Higher education 60, 2 (2010), 187-204.

[26] Xu Wang, Srinivasa Teja Talluri, Carolyn Rose, and Kenneth Koedinger. 2019. UpGrade: Sourcing Student Open-Ended Solutions to Create Scalable Learning Opportunities. In Proceedings of the Sixth (2019) ACM Conference on Learning @ Scale (L@S '19). ACM, New York, NY, USA, Article 17, 10 pages. DOI : http://dx.doi.org/10.1145/3330430.3333614

[27] Nesra Yannier, Kenneth R Koedinger, and Scott E Hudson. 2015. Learning from mixed-reality games: Is shaking a tablet as effective as physical observation?. In Proceedings of the 33rd Annual ACM Conference on Human Factors in Computing Systems. ACM, 1045-1054.

[28] Ken Zeichner. 2012. The turn once again toward practice-based teacher education. Journal of teacher education 63, 5 (2012), 376-382. 